\title{
Response Variability in Transcranial Direct Current Stimulation: Why Sex Matters
}

\author{
Thorsten Rudroff ${ }^{1,2 *}$, Craig D. Workman ${ }^{1}$, Alexandra C. Fietsam ${ }^{1}$ and John Kamholz ${ }^{2}$ \\ ${ }^{1}$ Department of Health and Human Physiology, University of lowa, lowa City, IA, United States, ${ }^{2}$ Department of Neurology, \\ Carver College of Medicine, University of lowa Hospitals and Clinics, lowa City, IA, United States
}

Keywords: transcranial direct current stimulation, sex differences, hormones, cranial bone, cortical excitability

\section{INTRODUCTION}

OPEN ACCESS

Edited by:

Alireza Mohammadi,

Baqiyatallah University of Medical

Sciences, Iran

Reviewed by:

Kyung Mook Choi,

Korea University, South Korea

Mo Chen,

University of Minnesota Twin Cities,

United States

Evangelia G. Chrysikou,

Drexel University, United States

${ }^{*}$ Correspondence:

Thorsten Rudroff

thorsten-rudroff@uiowa.edu

Specialty section:

This article was submitted to

Neuroimaging and Stimulation,

a section of the journal

Frontiers in Psychiatry

Received: 22 April 2020

Accepted: 08 June 2020

Published: 19 June 2020

Citation:

Rudroff $T$, Workman $C D$, Fietsam $A C$ and Kamholz J (2020) Response

Variability in Transcranial Direct

Current Stimulation:

Why Sex Matters.

Front. Psychiatry 11:585.

doi: 10.3389/fpsyt.2020.00585
The past 20 years have seen an increased interest in non-invasive brain stimulation, such as transcranial direct current stimulation (tDCS), transcranial alternating current stimulation (tACS), and transcranial random noise stimulation (tRNS) (1). In tRNS, an alternating current is applied to the scalp with a constantly changing frequency, while tACS oscillates a sinusoidal current at a chosen frequency to interact with the brain's natural cortical oscillations. Since the discovery that continuous weak direct current stimulation induces lasting excitability changes in the human motor cortex $(2,3)$, tDCS has been applied in various studies involving the induction and modulation of cortical neuroplasticity. It is generally accepted that anodal tDCS enhances cortical excitability (3) and cathodal tDCS decreases cortical excitability (inhibition) (4). However, this is an overly simplistic assumption and the effects of tDCS are likely much more complex.

The past 20 years have also witnessed a surge of findings concerning the influences of sex on many aspects of brain function and behavior, including emotion, memory, vision, hearing, facial processing, pain perception, navigation, neurotransmitter levels, and stress hormone action. The introduction of human brain-imaging techniques, like positron emission tomography (PET) and functional magnetic resonance imaging (fMRI), have heightened awareness of sex differences and revealed sex-related influences on brain functions that were previously thought to be similar between the sexes (5). For example, in 2006 Kuo et al. (6) suggested that sex differences pose a potential source of variability in cortical plastic changes from tDCS and should be addressed in cortical neuroplasticity manipulation studies in humans. However, in the intervening 15 years, the majority of tDCS studies have ignored biological sex differences. This neglect may account for some of the high inter-subject variability bemoaned by many tDCS investigators and has certainly delayed progress in the field. This is unfortunate because research into sex influences is vital to fully understanding the underlying mechanisms of non-invasive brain stimulation, especially for tDCS which has shown great variability (7-13). Indeed, approximately $50 \%$ of participants do not respond to tDCS and some even show effects contrary to the expected excitability response (e.g., inhibition from anodal tDCS and excitation from cathodal tDCS), evidenced by amplitude changes in motor evoked potentials (MEPs) elicited by transcranial magnetic stimulation (TMS) (14-16). Interestingly, Adenazato et al. (17) recently showed sex-related differences in cognitive Theory of Mind (ToM) abilities in healthy aging. Specifically, their findings showed that a single session of anodal tDCS over the medial prefrontal cortex led to significant slowing of reaction times in a communicative intention processing task in older women but not in men. 
The purpose of this opinion article is to emphasize the consideration of biological sex, in perspective of physiological and anatomical considerations, on tDCS outcomes and to provide reflections and directions for future studies in the field. The focus of this paper is on transcranial direct current stimulation (tDCS), although the discussed issues can be applied to other transcranial electrical stimulation methods.

\section{HORMONAL LEVELS AND NEUROTRANSMITTER BALANCES}

Hormonal levels fluctuate considerably more in women than in men and some studies exclude women from their research to reduce noise in their results (18). Men lack the cyclic fluctuation of sex hormones; testosterone and its metabolites, therefore, modulate cortical excitability similarly on different days. There are two main phases in the menstrual cycle of women: the follicular phase characterized by rising levels of estrogen and low levels of progesterone; and the luteal phase, which begins with ovulation and is accompanied by moderate levels of estrogen and high levels of progesterone. During the first follicular phase of menstruation (days 1-7), progesterone and estradiol levels are low and cortical excitation and inhibition from tDCS are less responsive. In the second follicular phase (days 7-14), estradiol increases while progesterone remains low, and excitability is enhanced and inhibition is reduced. In the first (days 14-21) and second luteal phases (days 21-28), when estradiol levels are moderate and progesterone levels are high, excitation is reduced and inhibition is enhanced (19-21). Thus, it appears, that progesterone drives the increase of cortical inhibition and estradiol enhances excitability. Additionally, measures of neurotransmitter levels important in tDCS responses (e.g., Gamma aminobutyric acid [GABA] and glutamate) support hormonal influences on cortical excitability. Using magnetic resonance spectroscopy (MRS), Epperson et al. (22) showed, in healthy women, that GABA concentrations in the primary visual cortex were lower in the luteal than in the follicular phase and that GABA was inversely correlated with both progesterone and estradiol levels. Similarly, another study found that glutamate concentrations in the medial frontal cortex were lower during the luteal than the follicular phase (23). Therefore, it is possible that assessing of the ratio of GABA/glutamate and estrogen/progesterone in future studies will confirm previous TMS findings that inhibition is increased during the luteal phase and reduced during the follicular phase, and excitation is reduced in the luteal phase and increased in the second follicular phase. Future tDCS studies that include women should carefully consider menstrual phases in their research designs, and women enrolled in repeated-session studies should be stimulated during the same phase of their menstrual cycle.

Supposing that an increase in cortical excitability is useful to enhance cognitive and motor function, negative consequences and non-optimal performances should be also noted.
Overexcitation of the cortex, for example in the second follicular phase (i.e., excessive release of glutamate) might result in excitotoxicity and cell death $(24,25)$. However, it is noted that there have been no instances of seizures induced by tDCS using intensities $\leq 4 \mathrm{~mA}$, which is considered safe for both men and women. Overexcitation likely depends on the targeted cortical region because the optimal excitation/inhibition (E/I) balance differs between brain areas. One location might exhibit overexcitation for a given set of tDCS parameters (e.g., intensity, duration, etc.) while others with different E/I levels, or an individual with lower excitation, may experience benefits (26). Indeed, the greater fatigability from $4 \mathrm{~mA}$ tDCS found in young women compared to young men by Workman et al. (27) could have been the result of overexcitation of the women. Excessive GABAergic inhibition also reduces neuronal output (28) and enhanced inhibition is correlated with higher network stability and reduced cortical plasticity (29).

A study by Stagg et al. (30) showed a significant correlation between the degree to which tDCS decreases GABA and a subject's ability to learn a novel task. Specifically, those who demonstrated a greater decrease in GABA from tDCS also showed greater enhancements in learning. Therefore, a balance in the E/I interaction might be necessary to improve the efficacy of information transfer in the brain $(31,32)$. For tDCS, this indicates a specific dose-response association that interrelates with pre-existing baseline levels, which are unknown to the investigator. In addition to other confounding factors, this interaction could clarify the observed differences in tDCS outcomes and the great variability common in tDCS studies.

\section{CORTICAL BONE STRUCTURE}

Another factor to consider is variations in skull structure/ composition between men and women. This is important because a study by Vöröslakos et al. (33) found that only a small portion of the transcranial current reached the brains of human cadavers. They estimated that the soft tissues (e.g., scalp, subcutaneous tissue, and muscle) shunted $\sim 50 \%$ of the current intensity and the skull shunted another 10-25\%. Additionally, Russell et al. (34) modeled current intensities (from 0.5, 1, to 2 $\mathrm{mA}$ tDCS) arriving at the cortex of men and women using different sex-specific skull characteristics derived from real MR images. Their results revealed interesting sex-specific variations, with their model suggesting that men would receive $\sim 45 \%$ more current at the cortex than women. The authors ascribed their results to the skull structure differences noted in the MRIs of men and women. Specifically, men had thicker skulls than women and the bone composition of the men was predominantly cancellous, while cortical bone was chiefly found in the women. Considering that cortical bone has a higher density than cancellous bone, the authors concluded that the denser cortical bone prevented more current from arriving at the cortex in the women. Consequently, possible differences in cortical bone density and cancellous bone thickness between men and women, and within each sex, should be considered in future tDCS study designs. 


\section{CHALLENGES, SOLUTIONS, AND FUTURE DIRECTIONS}

As noted above, some studies exclude women to control for the hormonal fluctuations and their effect on cortical excitation. Although this represents one potential solution, if the goal is to have an impact on the brain and behavior of the general population with tDCS, the correlation between the menstrual cycle and E/I must be explored further and women need to be included as participants. Hormonal levels measured from blood samples in women may provide indications of relative cortical excitability levels. However, more evidence is needed to validate the correlation between E/I and hormonal interactions. Another possible solution is to explicitly target the individual regional E/I balance by measuring GABA and glutamate levels, e.g., using MRS, and in so doing define the current polarity/parameters to optimize E/I. Although this method could be technically and practically challenging, such a study could carefully associate GABA and glutamate levels with other, more-easily measured individual characteristics (e.g., sex, age, hormone levels, etc.) to provide potential biomarkers of tDCS responsiveness.

For future research, it is particularly important that scientists plan for potential sex-related differences and that they choose their desired research populations with care to avoid unwanted noise in the data and/or, in extreme cases, the potential increased risk of seizure (35). This could be accomplished by accounting for these variables by pre-assessing E/I levels or activation patterns using neuroimaging methods (PET, fMRI, MRS), TMS, or at least behavioral patterns of performance. MRI or Computed Tomography (CT) could be also used to estimate cranial bone thickness and density and hormonal levels, especially in women, should be considered to avoid unwanted effects on tDCS cortical excitability. Specifically, systematic investigations to discover typical skull characteristics of men and women, and their association with cortical excitability, would help customize tDCS intensity by sex. tDCS should also be applied during specific phases of the menstrual cycle to

\section{REFERENCES}

1. Ruffini G, Wendling F, Merlet I, Molaee-Ardekani B, Mekonnen A, Salvador $\mathrm{R}$, et al. Transcranial current brain stimulation (tCS): models and technologies. IEEE Trans Neural Syst Rehabil Eng (2013) 21(3):333-45. doi: 10.1109/TNSRE.2012.2200046

2. Nitsche MA, Paulus W. Excitability changes induced in the human motor cortex by weak transcranial direct current stimulation. J Physiol (2000) 527:633-9. doi: 10.1111/j.1469-7793.2000.t01-1-00633.x

3. Nitsche MA, Paulus W. Sustained excitability elevations induced by transcranial DC motor cortex stimulation in humans. Neurology (2001) 57:1899-901. doi: 10.1212/WNL.57.10.1899

4. Nitsche MA, Nitsche MS, Klein CC, Tergau F, Rothwell JC, Paulus W. Level of action of cathodal DC polarization induced inhibition of the human motor cortex. Clin Neurophysiol (2003) 114:600-4. doi: 10.1016/S1388-2457(02)00412-1

5. Cahill L. Why sex matters for neuroscience. Nat Rev Neurosci|AOP (2006). published online 10 May. 7(6):477-84. doi: 10.1038/nrn1909

6. Kuo MF, Paulus W, Nitsche MA. Sex differences in cortical neuroplasticity in humans. Neuroreport (2006) 17:1703-7. doi: 10.1097/01.wnr.0000239955. $68319 . c 2$ optimize the effects of tDCS outcomes. For example, if tDCS is applied during the second follicular phase of the menstrual cycle, the intensity may need to be lower than in men to avoid overexcitation and create a positive outcome. Further individualization could then follow by measuring hormonal levels and bone composition of each individual and stimulating accordingly. This further strengthens the argument that tDCS should be customized for individual participants and discovering sex-related stimulation parameters would be a logical first step. It is noted that these sex differences are founded in biological variants inherent in men and women. Thus, future investigations should determine the biological sex of their subjects to control for the potential sources of variation discussed here.

The elucidation of ideal tDCS methodologies specific to each sex (e.g., low intensities for men, high intensities for women, or vice versa) constitutes a vital foundation for individualized tDCS application for participants. However, sex-related differences are only one of many issues (e.g. age, handedness, cognitive ability, neurological and psychiatric disorders, medications, recreational drugs, prior exposure to brain stimulation, electrode configurations, stimulation parameters, task dependency) suspected to contribute to the high variability in tDCS outcomes. Thus, it is questionable whether standardized tDCS applications are feasible, at least in the near future. Furthermore, it is virtually impossible to include all characteristics of every individual into clinical trial study designs to obtain homogenous samples, and tDCS tailored to individual participants is a more likely solution to response heterogeneity.

\section{AUTHOR CONTRIBUTIONS}

TR, CW, AF, and JK contributed to drafting the article and revising it critically for important intellectual content. All authors contributed to the article and approved the submitted version.

7. Horvath JC, Carter O, Forte JD. No significant effect of transcranial direct current stimulation (tDCS) found on simple motor reaction time comparing 15 different simulation protocols. Neuropsychologia (2016) 91:544-52. doi: 10.1016/j.neuropsychologia.2016.09.017

8. Horvath JC, Vogrin SJ, Carter O, Cook MJ, Forte JD. Effects of a common transcranial direct current stimulation (tDCS) protocol on motor evoked potentials found to be highly variable within individuals over 9 testing sessions. Exp Brain Res (2016) 234:2629-42. doi: 10.1007/s00221-016-4667-8

9. de Araújo AVL, Ribeiro FPG, Massetti T, Potter-Baker KA, Cortes M, Plow $\mathrm{EB}$, et al. Effectiveness of anodal transcranial direct current stimulation to improve muscle strength and motor functionality after incomplete spinal cord injury: a systematic review and meta-analysis. Spinal Cord (2020) 58(6):63546. doi: 10.1038/s41393-020-0438-2

10. Baharlouei H, Saba MA, Yazdi MJS, Jaberzadeh S. The effect of transcranial direct current stimulation on balance in healthy young and older adults: A systematic review of the literature. Clin Neurophysiol (2020) 50:119-31. doi: 10.1016/j.neucli.2020.01.006

11. Benoussi A, Pasqual-Leone A, Borroni B. Non-Invasive Cerebellar Stimulation in Neurodegenerative Ataxia: A Literature Review. Int J Mol Sci (2020) 21:1948. doi: 10.3390/ijms21061948 
12. Lloyd DM, Wittkopf PG, Arendsen LJ, Jones AKP. Is transcranial direct current stimulation (tDCS) effective for the treatment of pain in fibromyalgia? A systematic review and meta-analysis. J Pain (2020). doi: 10.1016/ j.jpain.2020.01.003

13. Ramger BC, Bader KA, Davies SP, Stewart DA, Ledbetter LS, Simon CB, et al. Effects of Non-Invasive Brain Stimulation on Clinical Pain Intensity and Experimental Pain Sensitivity Among Individuals with Central Post-Stroke Pain: A Systematic Review. J Pain Res (2019) 12:3319-29. doi: 10.2147/ JPR.S216081

14. Wiethoff S, Hamada M, Rothwell JC. Variability in response to transcranial direct current stimulation of the motor cortex. Brain Stimul (2014) 7(3):46875. doi: 10.1016/j.brs.2014.02.003

15. López Alonso V, Cheeran B, Río-Rodríguez D, Fernandez-Del-Olmo M. Inter-individual variability in response to non-invasive brain stimulation paradigms. Brain Stimul (2014) 7(3):372-80. doi: 10.1016/j.brs.2014. 02.004

16. Moliadze V, Atalay D, Antal A, Paulus W. Close to threshold transcranial electrical stimulation preferentially activates inhibitory networks before switching to excitation with higher intensities. Brain Stimul (2012) 5:50511. doi: 10.1016/j.brs.2011.11.004

17. Adenzato M, Manenti R, Gobbi E, Enrici I, Rusich D, Cotelli M. Aging, sex and cognitive Theory of Mind: a transcranial direct current stimulation study. Sci Rep (2019) 9:18064.

18. Krause B, Kadosh RC. Not all brains are created equal: the relevance of individual differences in responsiveness to transcranial electrical stimulation. Front Syst Neurosci (2014) 24. doi: 10.3389/fnsys.2014.00025

19. Inghilleri M, Conte A, Curra A, Frasca V, Lorenzano C, Berardelli A. Ovarian hormones and cortical excitability. An rTMS study in humans. Clin Neurophysiol (2004) 115:1063-8. doi: 10.1016/j.clinph.2003.12.003

20. Smith MJ, Adams LF, Schmidt PJ, Rubinow DR, Wassermann EM. Effects of ovarian hormones on human cortical excitability. Ann Neurol (2002) 51:599603. doi: $10.1002 /$ ana. 10180

21. Smith MJ, Keel JC, Greenberg BD, Adams LF, Schmidt PJ, Rubinow DA, et al. Menstrual cycle effects on cortical excitability. Neurology (1999) 53:2069-72. doi: 10.1212/WNL.53.9.2069

22. Epperson CN, Haga K, Mason GF, Sellers E, Gueorguieva R, Zhang W, et al. Cortical gamma-amino butyric acid levels across the menstrual cycle in healthy women and those with premenstrual dysphoric disorder: a proton magnetic resonance spectroscopy study. Arch Gen Psychiatry (2002) 59:851858. doi: 10.1001/archpsyc.59.9.851

23. Batra NA, Seres-Mailo J, Hanstock C, Seres P, Khudabux J, Bellavance F, et al. Proton magnetic resonance spectroscopy measurement of brain glutamate levels in premenstrual dysphoric disorder. Biol Psychiatry (2008) 63:1178-84. doi: 10.1016/j.biopsych.2007.10.007
24. Faden AI, Demediuk P, Panter SS, Vink R. The role of excitatory amino acids and NMDA receptors in traumatic brain injury. Science (1989) 244:798-800. doi: 10.1126/science.2567056

25. Belousov AB. Novel model for the mechanisms of glutamate-dependent excitotoxicity: role of neuronal gap junctions. Brain Res (2012) 1487:12330. doi: 10.1016/j.brainres.2012.05.063

26. Krause B, Marquez-Ruiz J, Cohen Kadosh R. The effect of transcranial direct current stimulation: a role for cortical excitation/inhibition balance? Front Hum Neurosci (2013) 7:602. doi: 10.3389/fnhum.2013.00602

27. Workman CD, Kamholz J, Rudroff T. Increased leg muscle fatigability during 2 $\mathrm{mA}$ and $4 \mathrm{~mA}$ transcranial direct current stimulation over the left motor cortex. Exp Brain Res (2020) 238(2):333-43. doi: 10.1007/s00221-019-05721-w

28. Mcdonnell MN, Orekhov Y, Ziemann U. Suppression of LTP-like plasticity in human motor cortex by the GABAB receptor agonist baclofen. Exp Brain Res (2007) 180:181-6. doi: 10.1007/s00221-006-0849-0

29. Hess G, Donoghue JP. Long-term potentiation of horizontal connections provides a mechanism to reorganize cortical motor maps. J Neurophysiol (1994) 71:2543-7. doi: 10.1152/jn.1994.71.6.2543

30. Stagg CJ, Bachtiar V, Johansen-Berg H. The role of GABA in human motor learning. Curr Biol (2011) 21:480-4. doi: 10.1016/j.cub.2011.01.069

31. Turrigiano GG, Nelson SB. Hebb and homeostasis in neuronal plasticity. Curr Opin Neurobiol (2000) 10:358-64. doi: 10.1016/S0959-4388(00)00091-X

32. Bavelier D, Levi DM, Li RW, Dan Y, Hensch TK. Removing brakes on adult brain plasticity: from molecular to behavioral interventions. J Neurosci (2010) 30:14964-71. doi: 10.1523/JNEUROSCI.4812-10.2010

33. Vöröslakos M, Takeuchi Y, Brinyiczki K, Zombori T, Oliva A, Fernández-Ruiz A, et al. Direct effects of transcranial electric stimulation on brain circuits in rats and humans. Nat Commun (2018) 9:483. doi: 10.1038/s41467-018-02928-3

34. Russell M, Goodman T, Wang Q, Groshong B, Lyeth BG. Gender Differences in Current Received during Transcranial Electrical Stimulation. Front Psychiatry (2014) 5:104. doi: 10.3389/fpsyt.2014.00104

35. Finocch C, Ferrari M. Female reproductive steroids and neuronal excitability. Neurol Sci (2011) 32(Suppl.1):S31-5. doi: 10.1007/s10072-011-0532-5

Conflict of Interest: The authors declare that the research was conducted in the absence of any commercial or financial relationships that could be construed as a potential conflict of interest.

Copyright $\odot 2020$ Rudroff, Workman, Fietsam and Kamholz. This is an open-access article distributed under the terms of the Creative Commons Attribution License (CC BY). The use, distribution or reproduction in other forums is permitted, provided the original author(s) and the copyright owner(s) are credited and that the original publication in this journal is cited, in accordance with accepted academic practice. No use, distribution or reproduction is permitted which does not comply with these terms. 